\title{
A Vector Play Model for Finite- Element Eddy-Current Analysis Using the Newton-Raphson Method
}

$\operatorname{AUTHOR}(S)$ :

Mitsuoka, Ryuhei; Mifune, Takeshi; Matsuo, Tetsuji; Kaido, Chikara

\section{CITATION:}

Mitsuoka, Ryuhei ... [et al]. A Vector Play Model for Finite-Element Eddy-Current Analysis Using the Newton-Raphson Method. IEEE Transactions on Magnetics 2013, 49(5): 16891692

\section{ISSUE DATE:}

2013-05

URL:

http://hdl.handle.net/2433/174350

\section{RIGHT:}

(c) 2013 IEEE. Personal use of this material is permitted. Permission from IEEE must be obtained for all other uses, in any current or future media, including reprinting/republishing this material for advertising or promotional purposes, creating new collective works, for resale or redistribution to servers or lists, or reuse of any copyrighted component of this work in other works.; この論文は出版社版でありません。引用の際には出版社版をご確認ご利用ください。; This is not the published version. Please cite only the published version. 


\title{
A Vector Play Model for Finite-Element Eddy-Current Analysis Using the Newton-Raphson Method
}

\author{
Ryuhei Mitsuoka $^{1}$, Takeshi Mifune ${ }^{1}$, Tetsuji Matsuo ${ }^{1}$, Member IEEE, and Chikara Kaido ${ }^{2}$ \\ ${ }^{1}$ Graduate School of Engineering, Kyoto University, Kyoto, 615-8510, Japan \\ ${ }^{2}$ Kitakyushu National College of Technology, Kitakyushu, 802-0985, Japan
}

The differentiation of the vector hysteretic function represented by a vector play model is discussed for efficient nonlinear electromagnetic field computation using the Newton-Raphson method. The combination of the nonlinear finite-element method and the vector play model achieves accurate representation of the AC anisotropic magnetic property of non-oriented silicon steel sheet under rotational magnetic flux conditions. The proposed method is successfully applied to the eddy-current analysis of iron-cored inductors excited by a current or voltage source.

Index Terms — differentiation, finite element method, Newton-Raphson method, anisotropic vector hysteresis.

\section{INTRODUCTION}

$\mathrm{C}$ ompact and high-powered electromagnetic machinery $C_{\text {requires a high magnetic flux density reaching saturation }}$ in the iron-core, where electromagnetic field analysis has to handle a strong nonlinearity due to the anisotropic vector hysteretic property. To represent the vector hysteretic property of iron-core materials, a number of vector hysteresis models have been developed such as the vector Preisach model [1], the vector Jiles-Atherton model [2], and the E \& S model [3]. Among them, the vector play model [4]-[6] is one of the most accurate and efficient models to represent the vector hysteretic property of silicon steel sheet and is expected to be applied in finite element electromagnetic field analysis.

However, generally the differentiation of the vector hysteretic function, which is required for the Newton-Raphson iteration, is not always easy. This paper discusses the differentiation of vector hysteretic function represented by the vector play model, where an equivalent representation is proposed for easy implementation of the differentiation procedure.

\section{A Vector Play Model for Finite-Element Analysis}

\section{A. The differentiation of the isotropic vector hysteretic} function represented by a play model

An isotropic vector play model [6] with an input of magnetic flux density $\boldsymbol{B}$ is given as:

$$
\begin{aligned}
& \boldsymbol{H}=\boldsymbol{P}(\boldsymbol{B})=\int_{0}^{B_{S}} \boldsymbol{f}\left(\zeta, \boldsymbol{p}_{\zeta}(\boldsymbol{B})\right) \mathrm{d} \zeta \\
& \boldsymbol{f}(\zeta, \boldsymbol{p})=f(\zeta, \mid \boldsymbol{p}) \frac{\boldsymbol{p}}{|\boldsymbol{p}|}
\end{aligned}
$$

where $f(\zeta, \boldsymbol{p})$ is a shape function, $B_{\mathrm{S}}$ is the saturation magnetic flux density, and $\boldsymbol{p}_{\zeta}$ is a vector play hysteron with radius $\zeta$. The hysteron $\boldsymbol{p}_{\zeta}$ is given as:

Manuscript received October 31, 2012 (date on which paper was submitted for review). Corresponding author: T. Matsuo (e-mail: tmatsuo@kuee.kyoto-u.ac.jp).

Digital Object Identifier inserted by IEEE

$$
\boldsymbol{p}_{\zeta}(\boldsymbol{B})=\left\{\begin{array}{cc}
\boldsymbol{p}_{\zeta}^{0} & \left(\left|\boldsymbol{B}-\boldsymbol{p}_{\zeta}^{0}\right|<\zeta\right) \\
\boldsymbol{B}-\zeta \frac{\boldsymbol{B}-\boldsymbol{p}_{\zeta}^{0}}{\left|\boldsymbol{B}-\boldsymbol{p}_{\zeta}^{0}\right|} & \left(\left|\boldsymbol{B}-\boldsymbol{p}_{\zeta}^{0}\right| \geq \zeta\right)
\end{array}\right.
$$

where $\boldsymbol{p}_{\zeta}^{0}$ is $\boldsymbol{p}_{\zeta}$ at the previous time-point. The NewtonRaphson procedure requires the differentiation of (1).

When $\left|\boldsymbol{B}-\boldsymbol{p}_{\zeta}^{0}\right|<\zeta, \partial \boldsymbol{p}_{\zeta} / \partial \boldsymbol{B}$ becomes 0 because $\boldsymbol{p}_{\zeta}=\boldsymbol{p}_{\zeta}^{0}$. When $\left|\boldsymbol{B}-\boldsymbol{p}_{\zeta}^{0}\right|>\zeta$, from (3) and:

$$
\frac{\partial\left|\boldsymbol{B}-\boldsymbol{p}_{\zeta}^{0}\right|}{\partial \boldsymbol{B}}=\frac{1}{\left|\boldsymbol{B}-\boldsymbol{p}_{\zeta}^{0}\right|}\left(\boldsymbol{B}-\boldsymbol{p}_{\zeta}^{0}\right)^{\mathrm{T}},
$$

$\partial \boldsymbol{p}_{\zeta} / \partial \boldsymbol{B}$ is obtained as:

$$
\frac{\partial \boldsymbol{p}_{\zeta}}{\partial \boldsymbol{B}}=\left(1-\frac{\zeta}{\left|\boldsymbol{B}-\boldsymbol{p}_{\zeta}^{0}\right|}\right) \mathbf{1}+\left(\frac{\zeta}{\left|\boldsymbol{B}-\boldsymbol{p}_{\zeta}^{0}\right|^{3}}\right)\left(\boldsymbol{B}-\boldsymbol{p}_{\zeta}^{0}\right)\left(\boldsymbol{B}-\boldsymbol{p}_{\zeta}^{0}\right)^{\mathrm{T}}
$$

where $\mathbf{1}$ is the unit matrix. The differentiation $\partial \boldsymbol{p}_{\zeta} / \partial \boldsymbol{B}$ is discontinuous when $\left|\boldsymbol{B}-\boldsymbol{p}_{\zeta}^{0}\right|=\zeta$.

From (2), (5) and:

$$
\frac{\partial\left|\boldsymbol{p}_{\zeta}\right|}{\partial \boldsymbol{B}}=\frac{\boldsymbol{p}_{\zeta}{ }^{\mathrm{T}}}{\left|\boldsymbol{p}_{\zeta}\right|} \frac{\partial \boldsymbol{p}_{\zeta}}{\partial \boldsymbol{B}},
$$

the differentiation of $\boldsymbol{f}$ is given as:

$$
\frac{\partial \boldsymbol{f}}{\partial \boldsymbol{B}}=\left[\left(\frac{\partial f}{\partial p}-\frac{f}{\left|\boldsymbol{p}_{\zeta}\right|}\right) \frac{1}{\left|\boldsymbol{p}_{\zeta}\right|^{2}} \boldsymbol{p}_{\zeta} \boldsymbol{p}_{\zeta}{ }^{\mathrm{T}}+\frac{f}{\left|\boldsymbol{p}_{\zeta}\right|} \mathbf{1}\right] \frac{\partial \boldsymbol{p}_{\zeta}}{\partial \boldsymbol{B}} .
$$

Because of the term $1 /\left|\boldsymbol{p}_{\zeta}\right|$, the differentiation of $\boldsymbol{f}$ becomes numerically difficult around $\boldsymbol{p}=0$. This occurs in the demagnetization state. To avoid this difficulty, we propose an equivalent form described by:

$$
\begin{aligned}
& \boldsymbol{f}(\zeta, \boldsymbol{p})=f_{0}\left(\zeta,|\boldsymbol{p}|^{2}\right) \boldsymbol{p} \\
& f_{0}(\zeta, q)=f_{0}\left(\zeta, p^{2}\right)=f(\zeta, p) / p
\end{aligned}
$$

where $q=p^{2}$. The differentiation of $\boldsymbol{f}$ simply becomes:

$$
\frac{\partial \boldsymbol{f}}{\partial \boldsymbol{B}}=\left(2 \frac{\partial f_{0}}{\partial q} \boldsymbol{p}_{\zeta} \boldsymbol{p}_{\zeta}{ }^{\mathrm{T}}+f_{0} \mathbf{1}\right) \frac{\partial \boldsymbol{p}_{\zeta}}{\partial \boldsymbol{B}}
$$

which does not require the term $1 /\left|\boldsymbol{p}_{\zeta}\right|$.

The differentiation of $\boldsymbol{P}$ is given as: 


$$
\frac{\partial \boldsymbol{P}}{\partial \boldsymbol{B}}=\int_{0}^{B_{S}} \frac{\partial \boldsymbol{f}}{\partial \boldsymbol{B}} \mathrm{d} \zeta \cdot
$$

Ref. [1] proposed a modified vector play model as:

$$
\boldsymbol{P}^{*}=\boldsymbol{P}_{\|}+r(B) \boldsymbol{P}_{\perp}
$$

where $r(B)$ is the ratio of the measured hysteresis loss to the simulated loss given by $\boldsymbol{P}$ for the rotational input with amplitude $B$, and $\boldsymbol{P}_{\|}$and $\boldsymbol{P}_{\perp}$ are the parallel and vertical components of $\boldsymbol{P}$ to $\boldsymbol{B}$, which are:

$$
\begin{aligned}
& \boldsymbol{P}_{\|}=\frac{(\boldsymbol{P} \cdot \boldsymbol{B})}{|\boldsymbol{B}|^{2}} \boldsymbol{B} \\
& \boldsymbol{P}_{\perp}=\boldsymbol{P}-\boldsymbol{P}_{\|}=\boldsymbol{P}-\frac{(\boldsymbol{P} \cdot \boldsymbol{B})}{|\boldsymbol{B}|^{2}} \boldsymbol{B} .
\end{aligned}
$$

Consequently, the differentiations of $\boldsymbol{P}_{\|}$and $\boldsymbol{P}_{\perp}$ are given by:

$$
\begin{aligned}
& \frac{\partial \boldsymbol{P}_{\|}}{\partial \boldsymbol{B}}=\frac{1}{|\boldsymbol{B}|^{2}} \boldsymbol{B}\left(\boldsymbol{B}^{\mathrm{T}} \frac{\partial \boldsymbol{P}}{\partial \boldsymbol{B}}+\boldsymbol{P}^{\mathrm{T}}\right)+\frac{(\boldsymbol{P} \cdot \boldsymbol{B})}{|\boldsymbol{B}|^{2}} \mathbf{1}-2 \frac{(\boldsymbol{P} \cdot \boldsymbol{B})}{|\boldsymbol{B}|^{4}} \boldsymbol{B B}^{\mathrm{T}} \\
& \frac{\partial \boldsymbol{P}_{\perp}}{\partial \boldsymbol{B}}=\frac{\partial \boldsymbol{P}}{\partial \boldsymbol{B}}-\frac{\partial \boldsymbol{P}_{\|}}{\partial \boldsymbol{B}} .
\end{aligned}
$$

The differentiation of $\boldsymbol{P}^{*}$ is obtained as:

$$
\frac{\partial \boldsymbol{P}^{*}}{\partial \boldsymbol{B}}=(1-r) \frac{\partial \boldsymbol{P}_{\|}}{\partial \boldsymbol{B}}+r \frac{\partial \boldsymbol{P}}{\partial \boldsymbol{B}}+\frac{1}{|\boldsymbol{B}|} \frac{\partial r}{\partial B} \boldsymbol{P}_{\perp} \boldsymbol{B}^{\mathrm{T}}
$$

Because of the term $1 /|\boldsymbol{B}|$, the differentiation of $\boldsymbol{P}^{*}$ becomes numerically difficult around $\boldsymbol{B}=0$. However, this difficulty is removed by fixing $r(B)$ to 1 around $\boldsymbol{B}=0$ so that $\boldsymbol{P}^{*}$ becomes $\boldsymbol{P}$.

The Jacobian matrices $\partial \boldsymbol{P} / \partial \boldsymbol{B}$ and $\partial \boldsymbol{P}^{*} / \partial \boldsymbol{B}$ are not symmetric. Consequently, the Newton-Raphson iteration requires the solution of a non-symmetric linear system.

\section{B. Computational examination}

The static hysteretic property of non-oriented (NO) silicon steel sheet (JIS:35A230) is now simulated. The discretized vector play model is given as:

$$
\begin{aligned}
\boldsymbol{H}=\boldsymbol{P}(\boldsymbol{B}) & =\Delta \zeta \sum_{n=1}^{N_{\mathrm{p}}} f\left(\zeta_{n},\left|\boldsymbol{p}_{\zeta n}(\boldsymbol{B})\right|\right) \frac{\boldsymbol{p}_{\zeta n}(\boldsymbol{B})}{\left|\boldsymbol{p}_{\zeta n}(\boldsymbol{B})\right|} \\
& =\Delta \zeta \sum_{n=1}^{N_{\mathrm{p}}} f_{0}\left(\zeta_{n},\left|\boldsymbol{p}_{\zeta n}(\boldsymbol{B})\right|^{2}\right) \boldsymbol{p}_{\zeta n}(\boldsymbol{B})
\end{aligned}
$$

where $\zeta_{n},=n \Delta \zeta, \Delta \zeta=B_{\mathrm{S}} / N_{\mathrm{p}}$, and $N_{\mathrm{p}}$ is the number of hysterons. Because of the limitation of magnetic property measurement, $B_{\mathrm{S}}$ and $N_{\mathrm{p}}$ are set to $1.7 \mathrm{~T}$ and 34 . Fig. 1 shows the value of shape function $f(\zeta, p) \Delta \zeta$ and $f_{0}(\zeta, p) \Delta \zeta$ with $\zeta=0$, $B_{\mathrm{S}} / 2$, where $f_{0}(\zeta, 0) \Delta \zeta$ is obtained by extrapolation. They have negative values when $\zeta>0$ because the input of this play model is not $\boldsymbol{H}$ but $\boldsymbol{B}$. Figs. 2 and 3 show the $B H$ loops and $\partial P_{x} / \partial B_{x}$ for the alternating sinusoidal input of $\boldsymbol{B}$ with amplitudes of $0.5,1.0 \mathrm{~T}$. The proposed form (8) gives almost the equivalent property to that represented by the conventional form (2). The shape function $f$ gives step-wise waveform of $\partial P_{x} / \partial B_{x}$ because $f$ is peace-wise linear, which almost agrees with $\partial P_{x} / \partial B_{x}$ given by the formulation using $f_{0}$.

\section{Anisotropic vector hysteresis model}

An anisotropic vector model has been proposed as [6]:

$$
\boldsymbol{P}_{\mathrm{an}}(\boldsymbol{B})=\boldsymbol{W}_{B}(\boldsymbol{B}) \boldsymbol{P}^{*}
$$

where $\boldsymbol{W}_{\mathrm{B}}$ is an anisotropy matrix for which the components are single-valued functions of $\boldsymbol{B}$. The differentiation of $\boldsymbol{P}_{\text {an }}$ is given as follows. The anisotropy matrix $\boldsymbol{W}_{B}(\boldsymbol{B})$ is given as:

$$
\boldsymbol{W}_{\boldsymbol{B}}(\boldsymbol{B})=\operatorname{diag}\left(w_{x}\left(B, \varphi_{B}\right), w_{y}\left(B, \varphi_{B}\right)\right)
$$

where $\varphi_{B}=\tan ^{-1}\left(B_{y} / B_{x}\right)$. The differentiation of $\varphi_{B}$ is:

$$
\frac{\partial \varphi_{B}}{\partial B_{x}}=-\frac{B_{y}}{\left(B_{x}{ }^{2}+B_{y}{ }^{2}\right)}, \frac{\partial \varphi_{B}}{\partial B_{y}}=\frac{B_{x}}{\left(B_{x}{ }^{2}+B_{y}{ }^{2}\right)} .
$$

Consequently, the differentiation of $\boldsymbol{W}_{\mathrm{B}}$ is obtained as:

$$
\begin{aligned}
\frac{\partial w_{x}\left(B, \varphi_{B}\right)}{\partial \boldsymbol{B}} & =\frac{\partial w_{x}}{\partial B} \frac{\partial B}{\partial \boldsymbol{B}}+\frac{\partial w_{x}}{\partial \varphi_{B}} \frac{\partial \varphi_{B}}{\partial \boldsymbol{B}} \\
& =\frac{\partial w_{x}}{\partial B} \frac{\boldsymbol{B}^{T}}{|\boldsymbol{B}|}+\frac{\partial w_{x}}{\partial \varphi_{B}} \frac{1}{|\boldsymbol{B}|^{2}}\left(-B_{y}, B_{x}\right) \\
\frac{\partial w_{y}\left(B, \varphi_{B}\right)}{\partial \boldsymbol{B}} & =\frac{\partial w_{y}}{\partial B} \frac{\boldsymbol{B}^{T}}{|\boldsymbol{B}|}+\frac{\partial w_{y}}{\partial \varphi_{B}} \frac{1}{|\boldsymbol{B}|^{2}}\left(-B_{y}, B_{x}\right) .
\end{aligned}
$$

Similarly, $w_{x}$ and $w_{y}$ are fixed to 1 around $\boldsymbol{B}=0$ to avoid using the term $1 /|\boldsymbol{B}|$.

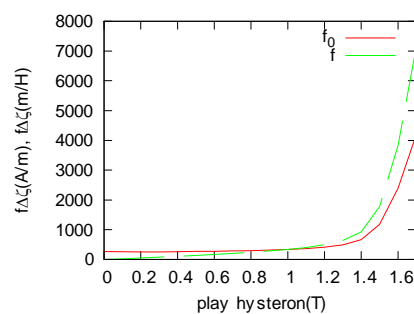

(a)

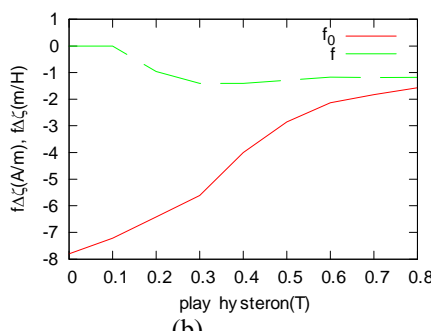

(b)
Fig. 1 Shape functions: (a) at $\zeta=0$, and (b) at $\zeta=B_{\mathrm{S}} / 2$.

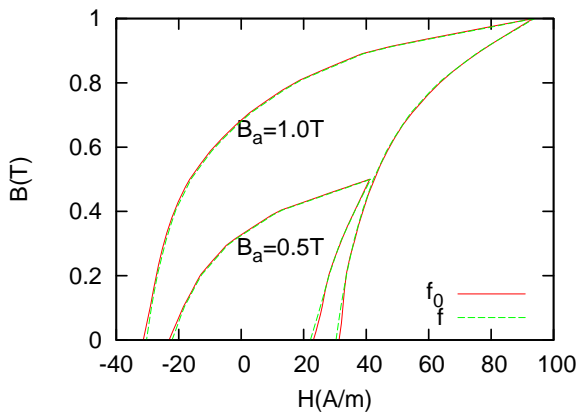

Fig. 2 Simulated static $B-H$ property of NO silicon steel.

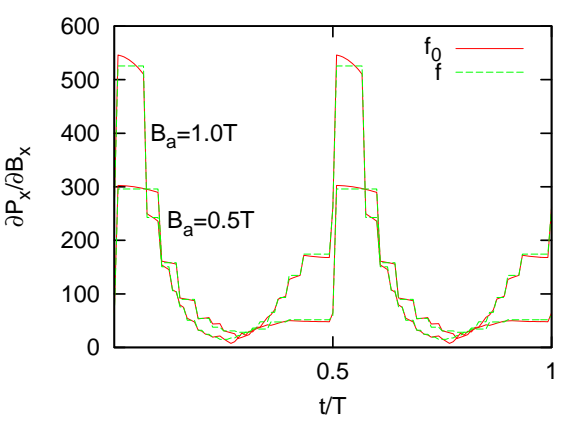

Fig. $3 \partial P_{x} / \partial B_{x}$ for a cycle corresponding to Fig. 2 ( $T$ : period.) 
The differentiation of $\boldsymbol{P}_{\text {an }}$ is obtained as:

$$
\frac{\partial \boldsymbol{P}_{\mathrm{an}}}{\partial \boldsymbol{B}}=\left(\begin{array}{l}
P_{x}^{*} \partial w_{x} / \partial \boldsymbol{B} \\
P_{y}^{*} \partial w_{y} / \partial \boldsymbol{B}
\end{array}\right)+\boldsymbol{W}_{B} \frac{\partial \boldsymbol{P}^{*}}{\partial \boldsymbol{B}} .
$$

\section{APPLICATION to Finite ELEMENT ANALysis}

A. Simulation of AC anisotropic vector hysteretic property of non-oriented silicon steel sheet.

Using the vector play model, Ref. [7] developed an accurate and efficient $\mathrm{AC}$ anisotropic vector hysteresis model for nonoriented steel sheets up to a frequency of $200 \mathrm{~Hz}$, where a dynamic term is added for the representation of the eddycurrent field. However, its representation at higher frequencies is not very accurate because it ignores the phase lag of the eddy-current field with respect to $\mathrm{d} B / \mathrm{d} t$. To describe the influence of the eddy-current field accurately, several hysteresis models include one-dimensional analysis along the sheet thickness direction [8], [9].

In this subsection, the one-dimensional finite element analysis is combined with the vector play model to represent the AC anisotropic vector hysteretic property of non-oriented silicon steel sheet.

The vector field in the steel sheet is described by the vector potential $\boldsymbol{A}=\left(A_{x}(z), A_{y}(z), 0\right)$, where $z$ is the coordinate along the direction of thickness. The governing equations are given as:

$$
\begin{aligned}
& -\partial H_{y}\left(B_{x}, B_{y}\right) / \partial \mathbf{z}=-\sigma \partial A_{x} / \partial t, \\
& \partial H_{x}\left(B_{x}, B_{y}\right) / \partial \mathbf{z}=-\sigma \partial A_{y} / \partial t \\
& B_{x}=-\partial A_{y} / \partial z, B_{y}=\partial A_{x} / \partial z
\end{aligned}
$$

where $\sigma$ is the conductivity. The anomalous eddy-current loss is ignored because it is small under large rotational flux conditions [7].

The rotational magnetic property of NO silicon steel sheet JIS: 35A230 is measured and simulated under the rotational magnetic flux condition. The sheet thickness and conductivity are $0.35 \mathrm{~mm}$ and $1.8 \times 10^{6} \mathrm{~S} / \mathrm{m}$. Its circular sample having slits at the periphery is excited by a stator of a single-phase, twopole induction motor [12]. A digital feedback waveform control achieves the circular loci of $\boldsymbol{B}$ with an error of less than $1 \%$.

Fig. 4 portrays the measured and simulated loci of the surface magnetic field vector at 200 and $500 \mathrm{~Hz}$ with an amplitude of $1-1.4 \mathrm{~T}$. The anisotropic rotational hysteretic property almost agrees with the measured one. A small discrepancy is observed between the measured and simulated loci mainly because the representation of anisotropy using (19) and (20) is too simple.

Combined with the line search method [10], the NewtonRaphson iteration converges successfully.

\section{B. Two-dimensional Finite Element Analysis}

The vector play model is implemented in a simple twodimensional finite-element analysis to confirm the numerical feasibility of the proposed model.
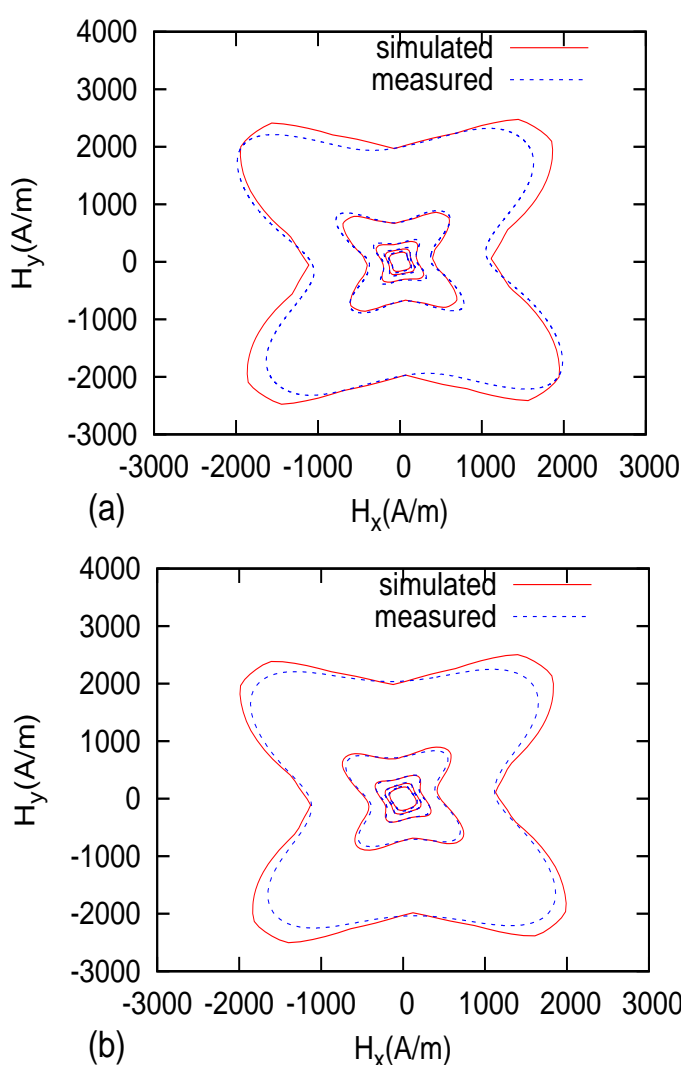

Fig. 4 Simulated AC anisotropic vector property of non-oriented silicon steel sheet under rotational magnetic flux with amplitude of 1 to $1.4 \mathrm{~T}$ : (a) at 200 $\mathrm{Hz}$, and (b) at $500 \mathrm{~Hz}$.

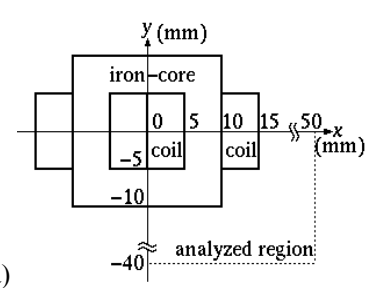

Fig. 5 Inductor analysis: (a) iron-cored inductor, and (b) exciting circuit.

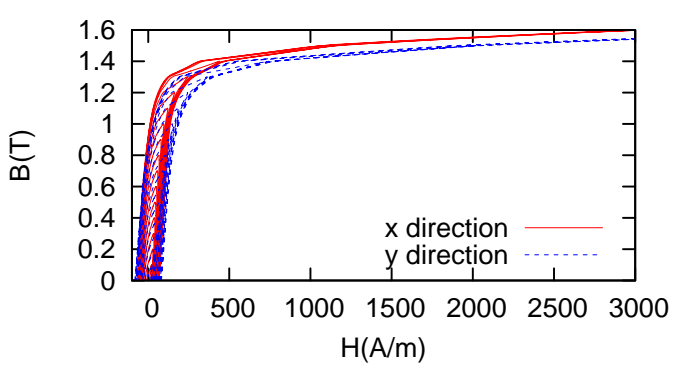

Fig. 6 Property of iron-core: (a) along $x$-direction, and (b) along $y$-direction.

The iron-cored inductor [11] shown in Fig. 5(a) is analyzed, where the virtual thickness $l_{z}$ is assumed along the $z$-direction. The iron-core is not laminated and its property is shown in Fig. 6. A quarter region, shown in Fig. 5(a), is analyzed with 6000 triangular elements, where the iron-core is divided into 600 equal elements. The excitation frequency is $50 \mathrm{~Hz}$, the number of turns is 400 and the conductivity of iron-core is $1 \times 10^{6} \mathrm{~S} / \mathrm{m}$. Fig. 7(a) shows the relationship between the current $I$ and the magnetic flux $\Phi / l_{z}$ using a sinusoidal current source with 
amplitude of 1.0, 2.0 and 4.0 A. Fig. 7(b) shows the $\Phi / l_{z}-I$ relationship when the inductor is excited by the voltage source as shown in Fig. $5(\mathrm{~b})$, where $E / l_{z}=1,1.3,1.6 \mathrm{kV} / \mathrm{m}$ and $R / l_{z}=$ $40 \Omega / \mathrm{m}$. The Newton-Raphson iteration assisted by the line search method converges successfully with 5 to 6 iterations on average, where a direct linear system solver is used.

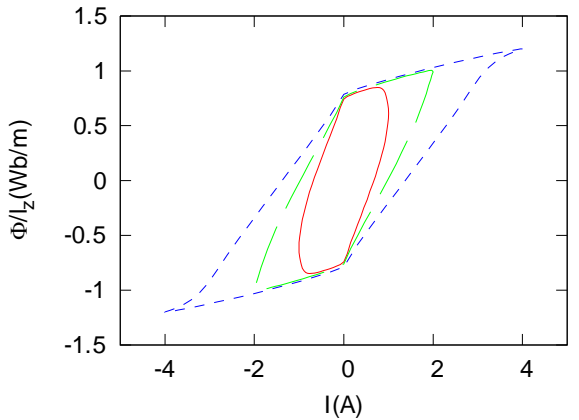

(a)

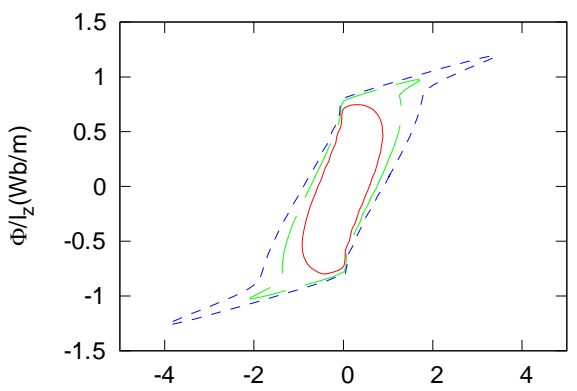

(b)

$\mathrm{I}(\mathrm{A})$

Fig. 7 Relationship between $I$ and $\Phi / l_{z}$ of iron-cored inductor: (a) excited by a sinusoidal current source, and (b) excited by a sinusoidal voltage source.

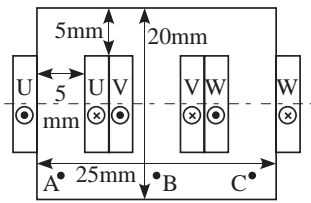

Fig. 8 Three-phase iron-cored Inductor.

Next, the three-phase iron-cored inductor shown in Fig. 8 is analyzed in a similar way to the single-phase case. The excitation frequency is $50 \mathrm{~Hz}$ and the number of turns is 100 . Fig. 9 portrays the loci of $\boldsymbol{B}$ and $\boldsymbol{H}$ at points A, B and C in Fig. 8 when the inductor is excited by a three-phase current source with amplitude of $1 \mathrm{~A}$. A rotational field is observed at point $\mathrm{B}$ The Newton-Raphson iteration assisted by the line search method converges successfully with about 6 iterations on average. Fig. 10 shows the convergence of the NewtonRaphson iterations for the first five time-steps, where a nearly quadratic convergence is achieved.

\section{CONCLUSIONS}

The differentiation scheme of a vector hysteretic function represented by the vector play model is improved for efficient nonlinear electromagnetic field computation using the Newton-Raphson method. The finite-element method is successfully applied to the eddy-current analysis of iron-cores with an anisotropic vector hysteretic property.
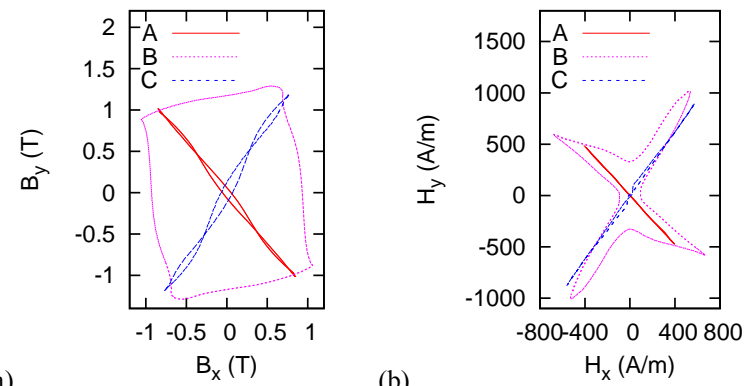

Fig. 9 Loci of $\boldsymbol{B}$ and $\boldsymbol{H}$ vectors: (a) $\boldsymbol{B}$ vector, and (b) $\boldsymbol{H}$ vector.

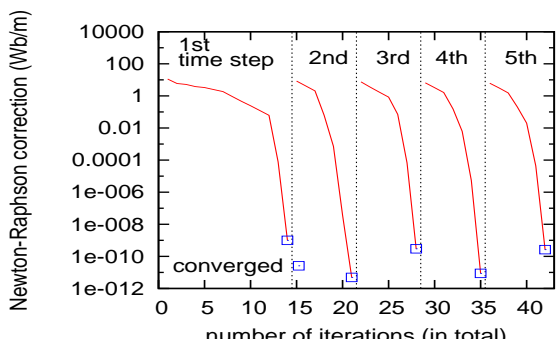

Fig. 10 Convergence of Newton-Raphson iterations, where the vertical axis means the norm of correction vector consisting of the node values of vector potential.

\section{ACKNOWLEDGMENT}

This work was supported in part by the Japan Society for the Promotion of Science, Grant-in-Aid for Scientific Research (C), Grant No. 23560328.

\section{REFERENCES}

[1] I.D. Mayergoyz, Mathematical Models of Hysteresis and their Applications, Elsevier, New York, 2003.

[2] J.V. Leite, N. Sadowski, P. Kuo-Peng, N.J. Batistela, J.P.A. Bastos, A.A de Espindola, "Inverse Jiles-Atherton vector hysteresis model," IEEE Trans. Magn., vol. 40, pp. 1769-1775, July 2004.

[3] T. Sato, M. Todaka and M. Enokizono, "Improvement of integrationtype dynamic E\\&S modeling," IEEE Trans. Magn., vol. 47, pp. 11261129, May 2011.

[4] A. Bergqvist, "Magnetic vector hysteresis model with dry friction-like pinning," Physica B, vol. 233, pp. 342-347, June 1997.

[5] M. d'Aquino, C. Serpico, C. Visone and A.A. Adly, "A new vector model of magnetic hysteresis based on a novel class of play hysterons," IEEE Trans. Magn., vol. 39, pp. 2537-2539, Sept. 2003.

[6] T. Matsuo, "Anisotropic vector hysteresis model using an isotropic vector play model, IEEE Trans. Magn., vol. 46, pp. 3041-3044, 2010.

[7] T. Matsuo, M. Miyamoto, "Dynamic and anisotropic vector hysteresis model based on isotropic vector play model for non-oriented silicon steel sheet, IEEE Trans. Magn., vol. 48, pp. 215-218, Feb. 2012.

[8] O, Bottauscio, M. Chiampi and D. Chiarabaglio, "Advanced model of laminated magnetic cores for two-dimensional field analysis," IEEE Trans. Magn., vol. 36, pp. 561-573, May 2000..

[9] R. Mitsuoka, T. Mifune, T. Matsuo, "Dynamic hysteresis modeling of silicon steel having nonuniform magnetic property, J. Appl. Phys., Vol. 111, Issue 7, 07D110, 2012.

[10] K. Fujiwara, Y. Okamoto, A. Kameari and A. Ahagon, "The NewtonRaphson method accelerated by using a line search -comparison between energy functional and residual minimization," IEEE Trans. Magn., vol. 41, pp.1724-1727, May 2005.

[11] T. Matsuo and M. Shimasaki, "Time-periodic finite element method for hysteretic eddy-current analysis," IEEE Trans. Magn., vol.38, pp. 549552, Mar. 2002.

[12] T. Matsuo, H. Hirao and M. Shimasaki, "Preliminary study of 2dimensional magnetic-property measurement of silicon steel sheet using stator of induction motor," Przeglad Elektrotechniczny, vol. 83, pp. 6769, 2007. 